\title{
HapticRein: Design and Development of an Interactive Haptic Rein for a Guidance Robot
}

\author{
Limin Zeng ${ }^{(\bowtie)}$, Björn Einert, Alexander Pitkin, and Gerhard Weber \\ Technische Universität Dresden, 01187 Dresden, Germany \\ \{limin. zeng, bjorn. einert, alexander.pitkin, \\ gerhard.weber\}@tu-dresden.de
}

\begin{abstract}
In daily life, a guide dog as a companion assists blind and visually impaired people (BVIP) to perform independent and safe journey. However, due to several reasons (e.g., cost and long-term training) only a few BVIP would own their guide dogs. To let much more BVIP would have accessible guidance services in unfamiliar public or private areas, we plan to develop an interactive guide dog robot prototype. In this paper, we present one of the most important components of a guidance robot for BVIP, an interactive haptic rein which consists of force-sensing sensors to control and balance the walking speed between BVIP and robots in a natural way, and vibrated actuators under fingers to acquire haptic information, e.g., turning left/right. The results of preliminary user studies indicated that the proposed haptic rein allow BVIP to control and communicate with a guidance robot via a natural haptic interaction.
\end{abstract}

Keywords: Haptic interaction - Guidance robot

Blind and visually impaired people

\section{Introduction}

For blind and visually impaired people (BVIP), a safe and independent indoor and outdoor mobility is an important connection with the society, such as going to office/hospital, shopping, and travelling. White canes and guide dogs have a high acceptance by BVIP, to assist them to detect obstacles and hazards while travelling and exploring the space. In order to overcome the limitations of those traditional travel aids (e.g., limited range, detection of hanging objects, and the expensive cost for owning a guide dog), in the last decades, a large number of electronic travel aids (ETAs) have been developed, like ultrasonic probe [1], vOICe system [2], 3DOD system [3] and Range-IT system [4].

Guidance robots have also been studied to provide guidance services for BVIP, with the help of various sensors [5,6]. As the development of robotic technologies and service robots in the last decade, an affordable and accessible guidance robot is becoming more and more promising in the near future. Those guidance robots would help BVIP not only detect obstacles and hazards, but also navigate themselves in familiar/unfamiliar areas, that is the traditional travel aids cannot offer. However, it is a 
challenge to communicate between BVIP and guided robots in time and in a non-visual way (e.g., auditory, haptic, and audio-haptic). Even auditory feedback (e.g., speech input/output) is a convenient approach, for BVIP it is important to hear environmental sounds. Furthermore, it is also a challenge to balance the walking speed between BVIP and robots.

In this paper, we present a novel haptic rein to support a non-visual commination between BVIP and guided robots. The haptic rein consists of a series of force-sensing sensors and vibrated actuators, where the force-sensing sensors are placed around the four sides of the handle of the haptic rein to detect push/pull movement for speeding up/down, and the vibrated actuators are placed on the bottom of the handle to deliver haptic messages to users' fingers. A preliminary user study indicated the proposed rein was effective to communicate and control a guidance robot to balance the walking speed, and some subjects commented it was a natural way to interact with a guidance robot.

\section{Related Work}

\subsection{Haptic/Tactile Interfaces for BVIP}

Haptic/tactile interaction as one of the most important non-visual user interfaces would provide accessible information for BVIP, in addition to auditory interfaces. In the last decades, a large number of accessible systems and devices which adopt haptic interfaces has been developed and evaluated. In general, the haptic interaction can be categorized into several types, such as vibrotactile interfaces $[4,7]$, pin-matrix display based haptic interfaces $[8,9]$, force-feedback based interfaces $[10,11]$ and electrostatic interfaces $[12,13]$. BVIP would acquire the direction and distance of the approaching obstacles via an array of actuators in a tactile belt [4], and learn their updated location on a tactile map [8]. However, due to the low resolution it is challenging to encode complex messages via haptic interfaces.

\subsection{Accessible Service Robots}

With the development of robotic technologies, various service robots have been produced in the last years. Accessible service robots are developed to assist people with speech needs in many different applications, by employing accessible user interfaces. Assistive robots for old people, as one of the primary application domains, would help them observe their health state [14], fetch objects [15], and other daily routines. Smart wheelchair robots enable wheelchair users to navigate intelligently [16], and robot arms allow upper-limb impaired people to interact with physical world flexible and independently [17]. Most of current accessible robots require that users should have considerable vision capability, nevertheless, there are a few accessible robots designed for visually impaired and blind people. 


\subsection{Haptic Interfaces for Guide Dog Robots}

A guide dog robot is a special kind of service robots, which assist BVIP to travel safely and independently, even in unfamiliar environment. Azenkot et al. suggested a guide dog robot should design the three primary accessible features: (1) summon the robot after entering the building, (2) three modes of assistance (Sighted Guide, Escort and Information Kiosk) while travelling, (3) receive information about the building's layout from the robot [18]. For the mode of Sighted Guide which emulates a human sighted guide, haptic interfaces are required to inform BVIP walking instructions (e.g., turning left/right). Table 1 compares some existing guide dog robots. On the one hand, some guide dog robots use canes $[6,19]$ and soft reins [20] to interact with users who need to perceive the changed direction of the canes or soft reins and follow them. On the other hand, a few systems investigate how to employ haptic sensors to communicate between users and robots. [21] develops an electrocutaneous handheld handle which connects with the robot via a wired cable, however, users have to train hardly to be familiar with the different pulse strength which encode the instructions, e.g., turning left/right and going closed to the robot. There is no study how to balance the walking speed between users and robots via a haptic interface.

Table 1. A comparison of some previous guide dog robots.

\begin{tabular}{l|l|l|l|l}
\hline Robots & Year & Main Features & (Haptic) Interface for users & Haptic Messages \\
\hline MELDOG & 1985 & $\begin{array}{l}\text { Obstacle avoidance } \\
\text { Map \& Route } \\
\text { Speech output } \\
\text { Haptic output }\end{array}$ & $\begin{array}{l}\text { An electrocutaneous } \\
\text { handheld handle } \\
\text { Wired rein }\end{array}$ & $\begin{array}{l}\text { Active haptic } \\
\text { output: } \\
\text { Turning } \\
\text { Warning }\end{array}$ \\
\hline GuideCane & 2001 & $\begin{array}{l}\text { Obstacle avoidance } \\
\text { Grid map } \\
\text { Control direction }\end{array}$ & $\begin{array}{l}\text { A hard cane with a } \\
\text { mini-joystick }\end{array}$ & $\begin{array}{l}\text { Passive haptic } \\
\text { output: following } \\
\text { the steering } \\
\text { angle of the } \\
\text { robot }\end{array}$ \\
\hline eyeDog & 2011 & $\begin{array}{l}\text { Obstacle avoidance } \\
\text { Autonomous navigation }\end{array}$ & A soft rein only & A hard cane \\
\hline $\begin{array}{l}\text { Co_robotic } \\
\text { Cane }\end{array}$ & 2016 & $\begin{array}{l}\text { Obstacle recognition } \\
\text { Speech interface }\end{array}$ & S &
\end{tabular}

\section{HapticRein System}

\subsection{System Overview}

The HapticRein system is developed based on a KUKA-Youbot 4-wheel robot platform, and a customized metal rein is attached to the robot platform (see Fig. 1). The length of the rein is $90 \mathrm{~cm}$ and the height of its handle to the floor is $88 \mathrm{~cm}$. The total weight of the robot is about $20 \mathrm{~kg}$, and maximal walking speed is about $0.8 \mathrm{~m} / \mathrm{s}$. A 2D laser LiDAR sensor is mounted in the front to detect obstacles. 


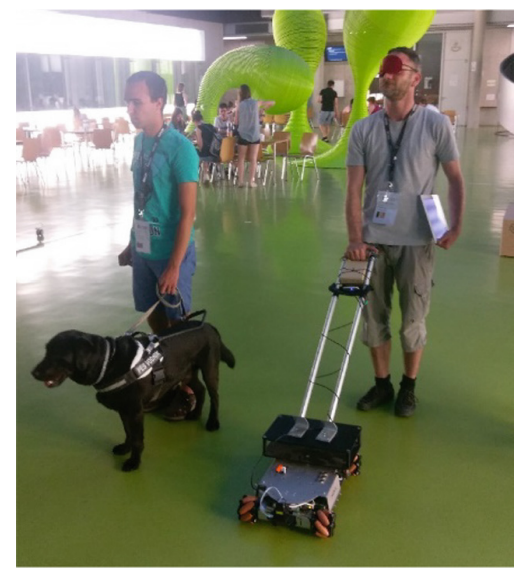

Fig. 1. The overview of the HapticRein system (left: a blind people with his own guide dog; right: a blindfolded people tested with the system).

\subsection{System Component of the HapticRein}

As illustrated in Fig. 2, there are several hardware components which are attached to the handle:

- Force-sensing sensors: 6 force-sensing sensors are placed around the four sides of the handle, to detect users' pull/push force value and press force.

- Vibrated actuators: 3 actuators are fixed at the bottom of the handle, where users' fingers can acquire haptic messages.

- An embedded board: An ARDUNIO UNO board is used to control the system.

- A thumb joystick: A thumb joystick is place on the top of the handle, and can easily make an input. Note that, the joystick is recognized as a baseline to compare with the force-based interaction.

\subsection{Human-Robot Haptic Interaction}

To support an interactive haptic interaction when communicating with and controlling the guide robot, the HapticRein system combines force-feedback and vibration feedback:

- Start walking: when a user grasp the handle with a considerable force detected by the Force Sensor 2 (on the top of the handle), the robot will start walking and its initial speed is $0.3 \mathrm{~m} / \mathrm{s}$;

- Speed up: to walk fast a user can push the handle, and the Force Sensor 1 (at the back of the handle) detects the increased force value, and the system speeds up. A user can continuously push the handle to speed up the robot, until a balance between them;

- Speed down: to walk slowly a user can pull the handle, and the Force Sensor 5 and Force Sensor 6 detect the reduced force value, when both of the force sensors detect 
the reduced force value and the value is bigger than a threshold, the robot will speed down;

- Soft stop: a user can continuously pull the handle to speed down the robot, until it stops;

- Hard stop: in some situations a user would need a quick or sudden stop, therefore, a hard stop is supported in the HapticRein when a user release the hand from the handle, and at the same time all force sensors can't detect input force value.

- Turning left/right: to turn the robot while walking a user can press the Force Sensor 3 and Force Sensor 4, and the press value will decide the turning angles (e.g., $30^{\circ}, 45^{\circ}, 60^{\circ}$ )

- Haptic messages: to deliver navigation messages (e.g., turning left/right and stop) via the 3 vibratos, a simple tactile pattern is designed.

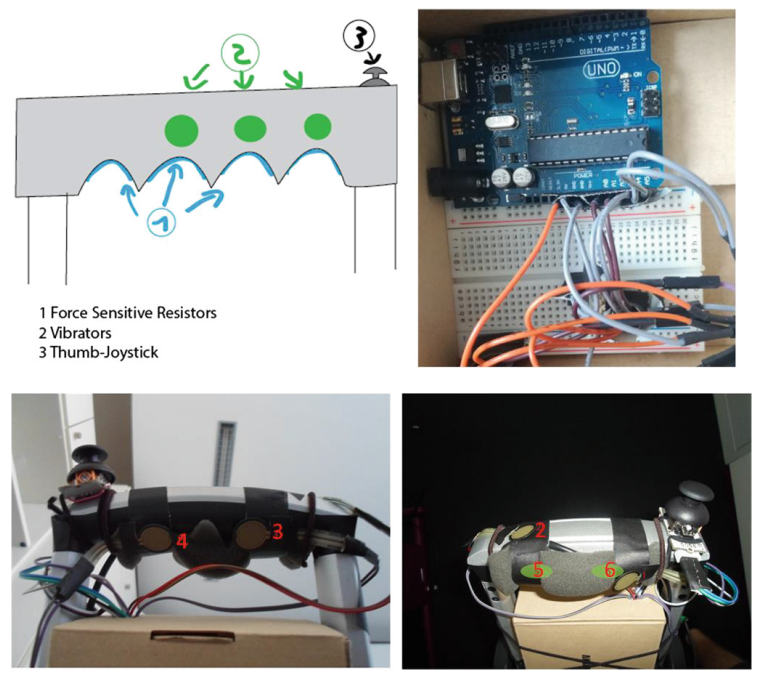

Fig. 2. The hardware components of the HapticRein system (top-left: an overview layout of the main sensors; top-right: the ARDUNIO board; bottom-left: bottom view of the handle; bottom-right: front view of the handle).

\section{Pilot Study}

A preliminary user study with five blindfolded subjects has been performed, and the subjects were in the average age of 25 years old. In addition to training how to use and control the robot system, the subjects were asked to finish a series of tasks, walking through a straight line and turning with a curve path (see Fig. 3). In the test, two different input tools were evaluated:

- Condition 1: using the mini-joystick

- Condition 2: using the proposed force handle 


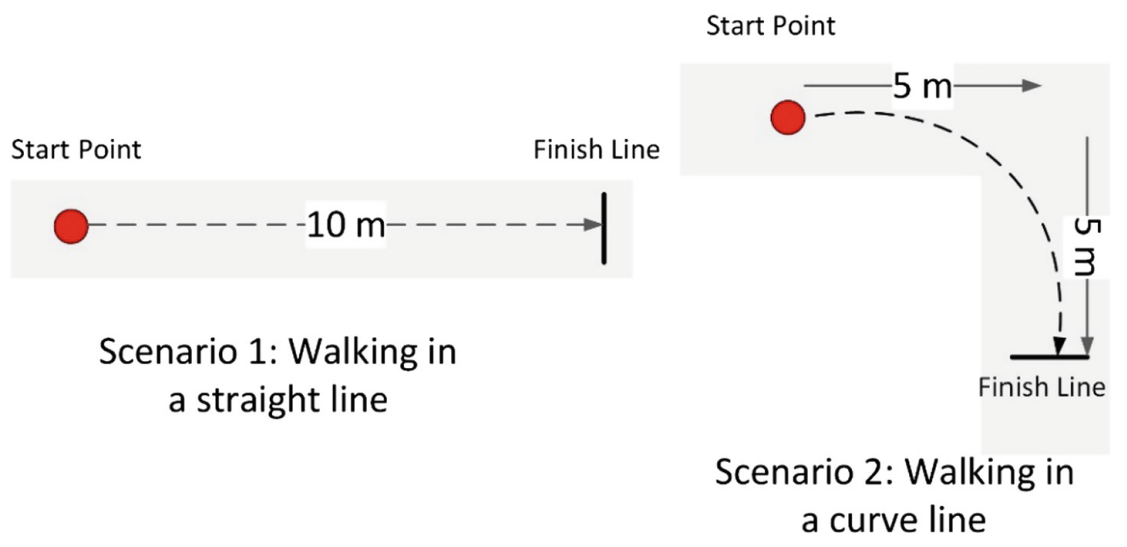

Fig. 3. The two test scenarios

The results indicated that the proposed force handle and the joystick both are effective to interact with the guidance robot, and the joystick was easier to learn. Three of the five subjects feedback that the force handle was intuitive and natural to control the walking speed. In particular, several subjects mentioned within the joystick solution users might make their thumb tried for a long distance walk.

Through the pilot study, several key issues have been found:

- The placement of the force sensors: The position of the force sensors when fixed in the handle is significant to gather precise force value. However, due to the different hand size our placement is not suitable for the subjects with small hands.

- The issue of sudden stop: We observed there were several sudden stops, and after analyzing the log data and evaluation videos we suspected the force value of Force Sensor 2 suddenly became zero that lead to sudden stops. One possible reason is while walking the finger contacted with the Force Sensor 2 was moved and left the sensor.

- The low maximum robot speed: Currently the maximum walking speed of our robot is $0.8 \mathrm{~m} / \mathrm{s}$ which is lower than the average walking speed with guide dogs $(1.05 \mathrm{~m} / \mathrm{s}$ [22]). In Scenario 1, when the robot quickly reached its maximum speed, the rear wheels were hang over the floor as the subjects walked faster and heavy push the handle.

\section{Conclusions and Future Work}

For blind and visually impaired people (BVIP), it is important to have a safe and independent indoor/outdoor travel, such as going to school/office, shopping and going to a doctor. Guide dogs would help BVIP avoid obstacles and hazards in their paths, however, it requires a long-term period to train a qualified guide dog and the expensive cost is not affordable for most of BVIP. As the development of service robots in the last decades, a guide dog robot as a promising mobility aid would offer affordable guidance for BVIP. 
In this paper, we present a guide dog robot prototype and propose a novel haptic rein to interact with guided robots and blind and visually impaired people. The haptic rein consists of force-sensing sensors and vibrated actuators to control the guidance robot (e.g., speed up/down and turning left/right) and acquire short haptic messages from the robot (e.g., turning left/right, and stop). A preliminary study with five blindfolded subjects has been conducted, and the results indicated the proposed haptic rein was effective and would offer an intuitive and natural way to control the guidance robot. Besides, via the study several issues have been found, such as the placement of the force sensors. In the coming future, we plan to invite blind and visually impaired people to evaluate the system, in addition to improving some design and technical features.

\section{References}

1. Kay, L.: An ultrasonic sensing probe as a mobility aid for the blind. Ultrasonics 2, 53-59 (1964)

2. Meijer, P.B.L.: An experimental system for auditory image representations. IEEE Trans. Biomed. Eng. 39, 112-121 (1992)

3. Zeng, L., Prescher, D., Weber, G.: Exploration and avoidance of surrounding obstacles for the visually impaired. In: Proceedings of the ACM ASSETS 2012, pp. 111-118 (2012)

4. Zeng, L., Simros, M., Weber, G.: Camera-based mobile electronic travel aids support for cognitive mapping of unknown spaces. In: Proceedings of the ACM MobileHCI 2017 (2017). Article No. 8

5. Tachi, S., Tanie, K., Komoriya, K., Abe, M.: Electrocutaneous communication in a guide dog robot (MELDOG). IEEE Trans. Biomed. Eng. BME-32, 461-469 (1985)

6. Ulrich, I., Borenstein, J.: The GuideCane-applying mobile robot technologies to assist the visually impaired. IEEE Trans. SMC 31, 131-136 (2001)

7. Zöllner, M., Huber, S., Jetter, H.-C., Reiterer, H.: NAVI - a proof-of-concept of a mobile navigational aid for visually impaired based on the microsoft kinect. In: Campos, P., Graham, N., Jorge, J., Nunes, N., Palanque, P., Winckler, M. (eds.) INTERACT 2011. LNCS, vol. 6949, pp. 584-587. Springer, Heidelberg (2011). https://doi.org/10.1007/978-3642-23768-3_88

8. Zeng, L., Weber, G.: Exploration of location-aware You-Are-Here maps on a pin-matrix display. IEEE Trans. Hum.-Mach. Syst. 46(1), 88-100 (2016)

9. Zeng, L., Miao, M., Weber, G.: Interactive audio-haptic map explorer on a tactile display. Interact. Comput. 27(4), 413-429 (2015)

10. Schneider, J., Strothotte, T.: Constructive exploration of spatial information by blind users. In: Proceedings of the 12th International ACM SIGACCESS Conference on Computers and Accessibility, Arlington, TX, USA, pp. 188-192 (2000)

11. Moustakas, K., Nikolakis, G., Kostopoulos, K., Tzovaras, D., Strintzis, M.G.: Haptic rendering of visual data for the visually impaired. IEEE MultiMed. 14(1), 62-72 (2007)

12. Tang, H., Beebe, D.J.: A microfabricated electrostatic haptic display for persons with visual impairments. IEEE Trans. Rehabil. Eng. 6(3), 241-248 (1998)

13. Xu, C., Israr, A., Poupyrev, I., Bau, O., Harrison, C.: Tactile display for the visually impaired using TeslaTouch. In: Proceedings of the CHI 2011 Extended Abstracts, pp. 317$322(2011)$ 
14. Brell, M., Meyer, J., Frenken, J., Hein, J.: A mobile robot for self-selected gait velocity assessments in assistive environments: a robotic driven approach to bring assistive technologies into established homes. In: Proceedings of the 3rd PETRA, pp. 15:1-15:8. ACM (2010)

15. Lee, M.K., Forlizzi, J., Rybski, P.E., Crabbe, F., Chung, W., Finkle, J., Glaser, E., Kiesler, S.: The snackbot: documenting the design of a robot for long-term human-robot interaction. In: Proceedings of the Human-Robot Interaction, pp. 7-14 (2009)

16. Wei, Z., Chen, W., Wang, J.: 3D semantic map-based shared control for smart wheelchair. In: Su, C.-Y., Rakheja, S., Liu, H. (eds.) ICIRA 2012. LNCS (LNAI), vol. 7507, pp. 41-51. Springer, Heidelberg (2012). https://doi.org/10.1007/978-3-642-33515-0_5

17. Graser, A., Heyer, T., Fotoohi, L., et al.: A supportive FRIEND at work: robotic workplace assistance for the disabled. IEEE Robot. Autom. Mag. 20(4), 148-159 (2013)

18. Azenkot, S., Feng, C., Cakmak, M.: Enabling building service robots to guide blind people a participatory design approach. In: Proceedings of the 11th ACM/IEEE International Conference on Human-Robot Interaction (HRI), Christchurch, pp. 3-10 (2016)

19. Ye, C., Hong, S., Qian, X., Wu, W.: Co-Robotic Cane: a new robotic navigation aid for the visually impaired. IEEE Syst. Man Cybern. Mag. 2(2), 33-42 (2016)

20. Galatas, G., McMurrough, C., Mariottini, G.L., Makedon, F.: Eyedog: an assistive-guide robot for the visually impaired. In: Proceedings of the 4th PETRA, pp. 58:1-58:8. ACM, New York (2011)

21. Tachi, S., Tanie, K., Komoriya, K., Abe, M.: Electrocutaneous communication in a guide dog robot (MELDOG). IEEE Trans. Biomed. Eng. BME-32(7), 461-469 (1985)

22. Clark-Carter, D.D., Heyes, A.D., Howarth, C.I.: The efficiency and walking speed of visually impaired people. Ergonomics 29(6), 779-789 (1986)

Open Access This chapter is licensed under the terms of the Creative Commons Attribution 4.0 International License (http://creativecommons.org/licenses/by/4.0/), which permits use, sharing, adaptation, distribution and reproduction in any medium or format, as long as you give appropriate credit to the original author(s) and the source, provide a link to the Creative Commons license and indicate if changes were made.

The images or other third party material in this chapter are included in the chapter's Creative Commons license, unless indicated otherwise in a credit line to the material. If material is not included in the chapter's Creative Commons license and your intended use is not permitted by statutory regulation or exceeds the permitted use, you will need to obtain permission directly from the copyright holder.

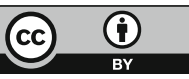

